\title{
The dual pattern of corticothalamic projection of the primary auditory cortex in macaque monkey.
}

Eric M. Rouiller and Caroline Durif

Unit of Physiology and Program in Neurosciences, Department of Medicine, Faculty of Sciences, University of Fribourg, Chemin du Musée 5, CH-1700 Fribourg, Switzerland.

Running title: Auditory cortex projections to the thalamus in monkey

Text pages: 11

Figures: 3 (3 in color)

Number of words in the main body text: 1458

Number of words in the abstract: 130

Keywords: temporal lobe; primate; auditory thalamus; anterograde tracing; axon terminal; medial geniculate body. 


\section{Abstract}

The distribution and terminal morphology of the corticothalamic projection originating from the primary auditory cortex (A1) were established in a macaque monkey, using the anterograde (and retrograde) tracer Biotinylated Dextran Amine. A dense corticothalamic projection from A1 was found in the ventral (vMGB) and dorsal (dMGB) divisions of the medial geniculate body and, to a lesser extent, in the medial division (mMGB), the posterior thalamic nucleus (PO) and the suprageniculate nucleus (SG). Most terminal boutons were small $(<1 \mu \mathrm{m})$, except some large boutons $(2-6 \mu \mathrm{m})$ located in PO and VMGB. The data demonstrate that the corticothalamic projection from A1 in primate consists of two types of terminals (small and giant endings) in line with previous observations in rat and cat. Retrogradely labeled thalamocortical neurons formed clusters generally overlapping the corticothalamic terminal fields. 
The application of modern anterograde tracers (biocytin, phaseolus vulgaris-leucoagglutinin or dextrans) led to the description of two types of corticothalamic (CT) terminals, the small $(<2 \mu \mathrm{m}$ in diameter) and giant endings $(2-6 \mu \mathrm{m})$. This dual pattern of CT termination is systematic across species and systems [16]. The major type of CT terminals consists of small endings, whereas the giant endings are far less numerous, forming spatially restricted territories. In the auditory system, the dual pattern of CT projection was found in rat [14] and cat $[1,9,20]$. The main CT projection formed by small endings terminates predominantly in the ventral division of the medial geniculate body (VMGB), whereas the CT projection formed by giant endings is in its dorsal division (dMGB). In contrast to the somatosensory, visual and motor systems in which the dual pattern of CT projection has been demonstrated in primates $[3,13,15,17]$, data are lacking in monkeys for the CT projection originating from the primary auditory cortex (A1). To fill this gap, the present study aimed at establishing the detailed morphology of CT endings in the monkey using the tracer Biotinylated Dextran Amine (BDA).

The methods of functional investigations of A1 in monkeys were described in detail elsewhere [4] and were conducted in accordance with the Guide for the Care and Use of Laboratory Animals (ISBN 0-309-05377-3; 1996) and approved by local veterinary authorities. The reciprocal projections between $\mathrm{A} 1$ and the thalamus were assessed in a macaque monkey (Macaca mulatta; $8 \mathrm{Kg}$ body weight). For an acute electrophysiological recording session (about 5-6 hours), the animal was pre-anesthetized with ketamine (5 mg/kg, i.m.), treated with the analgesic Carprofen (Rimadyl; $4 \mathrm{mg} / \mathrm{kg}$, s.c.), deeply anesthetized with propofol (3 ml/kg/hour, i.v.) and placed in a stereotaxic frame. The skull was opened above the parietal lobe to reach the left auditory cortex by means of vertical electrode penetrations [4] and using stereotaxic coordinates [11]. The propofol infusion was then interrupted and anesthesia was maintained for the rest of the recording session by repetitive (every $45 \mathrm{~min}$ ) i.m. injections of a mixture of ketamine ( $3 \mathrm{mg} / \mathrm{kg})$ and xylazine (1.5 $\mathrm{mg} / \mathrm{kg}$ ). Acoustic stimuli consisted of noise bursts, tone bursts or tone sweeps [4]. A few (4-5) electrode penetrations at various locations as well as landmarks derived from previous electrophysiological experiments in two other monkeys [4] were sufficient to locate A1 in this 
particular animal. Using a syringe (Hamilton; $10 \mu \mathrm{l})$, a total volume of $2 \mu \mathrm{l}$ of BDA ( $5 \%$ in saline) was injected in A1. The animal was treated several days with Carprofen and an antibiotic (Ampiciline $10 \% ; 30 \mathrm{mg} / \mathrm{kg}$, s.c.). After the survival period (10 days), an overdose of pentobarbital (i.p., 90 $\mathrm{mg} / \mathrm{kg}$ ) was given, followed by transcardiac perfusion with saline, $4 \%$ paraformaldehyde in $0.1 \mathrm{M}$ phosphate buffer $(\mathrm{pH} 7.4)$ and solutions of sucrose $(10 \%, 20 \%$ and $30 \%)$. Frozen sections of the brain were cut in the frontal plane $(50 \mu \mathrm{m})$ and collected in 8 series in phosphate buffer $0.1 \mathrm{M}(\mathrm{pH}$ 7.4), before processing for Nissl-staining, or visualization of BDA [17]. Sections of interest were plotted as previously described [15] or using the software Neurolucida (MicroBrightField, Colchester, VT, USA).

The BDA injection site in A1 is illustrated in Figure 1, covering the six cortical layers. Transposed to the atlas of the monkey brain [11], the injection site extends between the stereotaxic rostrocaudal coordinates 6.3 to $9.5 \mathrm{~mm}$, in the auditory koniocortex (areas AKL and AKM). The injection site is thus restricted to the area A1, located between the rostrocaudal coordinates 4 and $14 \mathrm{~mm}[5] . A$ typical CT axonal terminal field formed by small endings is depicted in Figure 2A. Since BDA is also transported retrogradely, most parts of the terminal fields formed by small endings exhibited some retrogradely labeled thalamocortical (TC) neurons. The main CT terminal field is located in VMGB and dMGB (Figs. 2A and 3) and, in addition, spreads medially to the suprageniculate nucleus (SG) and the medial division of the medial geniculate body (mMGB). Small and giant CT endings are shown in Figure 2B-C. Giant endings appear as spherical vesicles forming large boutons en passant and boutons terminaux. As shown previously [15], the diameters' range of giant endings $(2-6 \mu \mathrm{m})$ is separate from that of small endings $(<2 \mu \mathrm{m})$. Much more numerous, the small endings were seen predominantly in VMGB, as well as in AMGB, SG, mMGB (Fig. 3) and the reticular nucleus of the thalamus. Giant endings were much fewer, restricted to small territories in the posterior nucleus of the thalamus (PO), in VMGB and at the limit between VMGB and dMGB (Fig. 3). The giant endings are located midway along the rostrocaudal extent of the MGB. BDA being transported antero- and retro-gradely, one may argue that giant endings represent labeled collaterals of retrogradely stained TC neurons. This possibility is unlikely for at least two reasons. BDA retrograde labeling is clearly less dense than anterograde labeling, thus the axon and 
dendrites of TC neurons were lightly labeled. Giant endings were densely stained, typical of the anterograde labeling. Second, giant endings were also observed in zones devoid of stained TC neurons. Concerning the BDA retrograde labeling (Fig. 3), the majority of TC labeled neurons were in the main $C T$ terminal field in VMGB and $\mathrm{dMGB}$, but were also present in smaller CT terminal fields, in vMGB, mMGB and SG. Finally, a few isolated TC labeled neurons were observed outside CT terminal fields, in vMGB, mMGB and at the medial limit of SG.

As a result of BDA injection in A1 in the monkey, a main cluster of anterograde and retrograde labeling was observed in VMGB and AMGB (Figs. 2A and 3), in line with previous observations based on retrograde- $[6,8,12]$ and anterograde-tracing data [10]. The presence of labeling, but to a lesser extent, in $\mathrm{mMGB}, \mathrm{SG}$ and $\mathrm{PO}$ is also consistent with previous reports $[8,12]$. Besides confirmatory data about the topology of the TC and CT projections, the original contribution of the present study is to extend to the primate the dual pattern of arrangement of the CT projection from A1, consisting of small and giant endings, as previously established for rat and cat $[1,9,14,20]$. Such distinction of terminal endings escaped detection in a previous study in the monkey [10], because radio-labeled amino-acids did not allow the visualization of individual axonal endings. A further application of BDA, due to the yield of both anterograde and retrograde labeling for the same injection site, is to assess the degree of reciprocity of the TC and CT projections. The data illustrated in Figures 2 and 3 show that some restricted CT terminal territories are devoid of TC neurons, whereas restricted territories of the MGB with TC neurons are deprived of CT terminal fields. Nevertheless, there is evidence for a large and predominant overlap of the CT terminal fields with the clusters of retrogradely labeled TC neurons, in line with observations for A1 in cat [19].

It has been established that the giant endings arise from layer $\mathrm{V}$ CT neurons and small endings from layer VI CT neurons $[2,3,7,9,18]$. Such laminar distinction could not be assessed here because our BDA injection covered both layers $\mathrm{V}$ and VI. However, the presence of a few small endings along the same axonal branches giving rise to giant endings (Fig. 2C) suggests that layer V CT neurons can also produce small endings. The observation of small and giant endings along the same axonal branches is consistent with previous observations [14,15,17]. 
The presence of giant endings in PO (Fig. 3) is consistent with a previous report of such endings in POI in the cat [1]. The cluster of giant endings in VMGB (Fig. 3) may be interpreted as an interspecies difference with cat and rat, in which the giant endings were found mostly in dMGB $[1,14]$. However, in the later species, the giant endings were in the most ventral part of dMGB, if not in VMGB for a few of them [1]. In the monkey, the giant endings in VMGB are located dorsally near the border with $\mathrm{dMGB}$, or even in $\mathrm{dMGB}$ itself (Fig. 3). One can conclude that the giant endings occupy roughly the same territory (ventral dMGB - dorsal vMGB) in rat, cat and monkey and therefore appear to transgress the $\mathrm{VMGB/dMGB}$ border.

As discussed in details elsewhere $[16,17]$, the functional meaning of the dual pattern of CT projection is that small endings represent an anatomical support for a feedback projection, allowing the cerebral cortex to exert a descending control onto the thalamus. In contrast, giant endings correspond to a feedforward connection system, through which fast and secure transmission can go from one cortical area to another cortical region via the thalamus. Further experiments are needed in order to investigate whether non-primary auditory cortical areas in the monkey (belt and parabelt areas; [5]) also exhibit the same dual pattern of CT connection. 


\section{Acknowledgements:}

We thank V. Moret, C. Roulin, and F. Tinguely for their technical assistance for histology, J. Corpataux and B. Morandi for animal care and surgical assistance, A. Gaillard and B. Aebischer for mechanics and electronics, L. Monney for computer sciences. 


\section{References}

[1] Bajo VM, Rouiller EM, Welker E, Clarke S, Villa AEP, de Ribaupierre Y, de Ribaupierre F Morphology and spatial distribution of corticothalamic terminals originating from the cat auditory cortex. Hearing Res. 83 (1995) 161-174.

[2] Bourassa J, Pinault D, Deschênes M. Corticothalamic projections from the cortical barrel field to the somatosensory thalamus in rats: A single-fibre study using biocytin as an anterograde tracer. Eur. J. Neurosci. 7 (1995) 19-30.

[3] Darian-Smith C, Tan A, Edwards S. Comparing thalamocortical and corticothalamic microstructure and spatial reciprocity in the macaque ventral posterolateral nucleus (VPLc) and medial pulvinar. J. Comp. Neurol. 410 (1999) 211-234.

[4] Durif C, Jouffrais C, Rouiller EM Single unit responses in the auditory cortex of monkeys performing a conditional acoustico-motor task. Exp. Brain Res. 153 (2003) 614-627.

[5] Hackett TA, Stepniewska I, Kaas JH Subdivisions of auditory cortex and ipsilateral cortical connections of the parabelt auditory cortex in macaque monkeys. J. Comp. Neurol. 394 (1998) 475-495

[6] Hashikawa T, Rausell E, Molinari M, Jones EG Parvalbumin- and calbindin-containing neurons in the monkey medial geniculate complex: differential distribution and cortical layer specific projections. Brain Res. 544 (1991) 335-341.

[7] Kakei S, Na J, Shinoda Y. Thalamic terminal morphology and distribution of single corticothalamic axons originating from layers 5 and 6 of the cat motor cortex. J. Comp. Neurol. 437 (2001) 170-185.

[8] Morel A, Garraghty PE, Kaas JH Tonotopic organization, architectonic fields, and connections of auditory cortex in macaque monkeys. J. Comp. Neurol. 335 (1993) 437-459.

[9] Ojima $\mathrm{H}$. Terminal morphology and distribution of corticothalamic fibers originating from layers 5 and 6 of cat primary auditory cortex. Cereb. Cortex 4 (1994) 646-663.

[10] Pandya DN, Rosene DL, Doolittle AM Corticothalamic connections of auditory-related areas of the temporal lobe in rhesus monkey. J. Comp. Neurol. 345 (1994) 447-471.

[11] Paxinos G, Huang XF, Toga AW The rhesus monkey brain in stereotaxic coordinates. London; San Diego: Academic Press, 2000, pp. 1-165.

[12] Rauschecker JP, Tian B, Pons T, Mishkin M Serial and parallel processing in rhesus monkey auditory cortex. J. Comp. Neurol. 382 (1997) 89-103.

[13] Rockland KS. Two types of corticopulvinar terminations: round (type 2) and elongate (type 1). J. Comp. Neurol. 368 (1996) 57-87.

[14] Rouiller EM, Welker E Morphology of corticothalamic terminals arising from the auditory cortex of the rat: A phaseolus vulgaris-leucoagglutinin (PHA-L) tracing study. Hearing Res. 56 (1991) 179-190.

[15] Rouiller EM, Tanné J, Moret V, Kermadi I, Boussaoud D, Welker E. Dual morphology and topography of the corticothalamic terminals originating from the primary, supplementary motor, and dorsal premotor cortical areas in macaque monkeys. J. Comp. Neurol. 396 (1998) 169-185. 
[16] Rouiller EM, Welker E. A comparative analysis of the morphology of corticothalamic projections in mammals. Brain Res. Bull. 53 (2000) 727-741.

[17] Rouiller EM, Wannier T, Morel A The dual pattern of corticothalamic projection of the premotor cortex in macaque monkeys. Thalamus and Related Systems 2 (2003) 189-197.

[18] Schwartz ML, Dekker JJ, Goldman-Rakic PS. Dual mode of corticothalamic synaptic termination in the mediodorsal nucleus of the rhesus monkey. J. Comp. Neurol. 309 (1991) 289-304.

[19] Winer JA, Larue DT Patterns of reciprocity in auditory thalamocortical and corticothalamic connections: study with horseradish peroxidase and autoradiographic methods in the rat medial geniculate body. J. Comp. Neurol. 257 (1987) 282-315.

[20] Winer JA, Larue DT, Huang CL Two systems of giant axon terminals in the cat medial geniculate body :convergence of cortical and GABAergic inputs. J. Comp. Neurol. 413 (1999) 181-197. 


\section{Legends to Figures}

\section{Figure 1}

Illustration of the BDA injection site in A1. On the left (top), a lateral view of the left hemisphere of the monkey brain shows the plane and level of sectioning and a corresponding frontal section of the left hemisphere with a rectangle delineating $A 1$ in the ventral bank of the lateral fissure (If). $\mathrm{CE}=$ central sulcus; $\mathrm{GI}=$ insular granular cortex; ips=intraparietal sulcus; $\mathrm{S} 2=$ secondary somatosensory area; 1,2 and $3 b=$ subareas of the primary somatosensory area. In the right column, two photomicrogaphs illustrate the injection site at two rostrocaudal levels, separated by $1.6 \mathrm{~mm}$. The top panel illustrates the injection site covering upper cortical layers in A1 caudally, whereas the bottom panel illustrates the spread of the injection site to deep cortical layers more rostrally. On the bottom left, a Nissl-stained section taken in the middle of the injection site shows the mark left by the syringe in intermediate cortical layers. Scale bars $=1 \mathrm{~mm}$.

\section{Figure 2}

Photomicrographs illustrating the BDA labeled CT terminal fields in the medial geniculate body (MGB). Panel A shows the main and densest CT terminal field located in VMGB and AMGB (brown halo). The dark brown spots are retrogradely labeled TC neurons. Panels $B$ is a high magnification of a portion of the same main CT terminal field in VMGB showing retrogradely labeled TC neurons (open arrows) as well as small CT endings (few of them pointed by arrows). Panel C is a photomicrograph showing a small CT terminal field in PO, comprising both small endings (open arrows) and giant endings (arrows). Scale bar for panels $B$ and $C=50 \mu \mathrm{m}$.

\section{Figure 3}

Reconstruction of three individual frontal sections of the MGB showing the distribution of BDA labeled-CT terminal fields, -TC neurons and -giant CT endings. Sections were arranged from rostral (left) to caudal (right). 


\title{
List of abbreviations
}

\author{
A1 = primary auditory cortex \\ $\mathrm{BDA}=$ biotinylated dextran amine \\ $\mathrm{CT}=$ corticothalamic \\ $\mathrm{dMGB}=$ dorsal division of MGB \\ $\mathrm{LGN}=$ lateral geniculate nucleus \\ MGB = medial geniculate body \\ $\mathrm{mMGB}=$ medial division of MGB \\ $\mathrm{PO}=$ posterior nucleus of the thalamus \\ $\mathrm{Pul}=$ pulvinar nucleus \\ SG = suprageniculate nucleus \\ $\mathrm{TC}=$ thalamocortical \\ VMGB = ventral division of MGB
}


Fig. 1

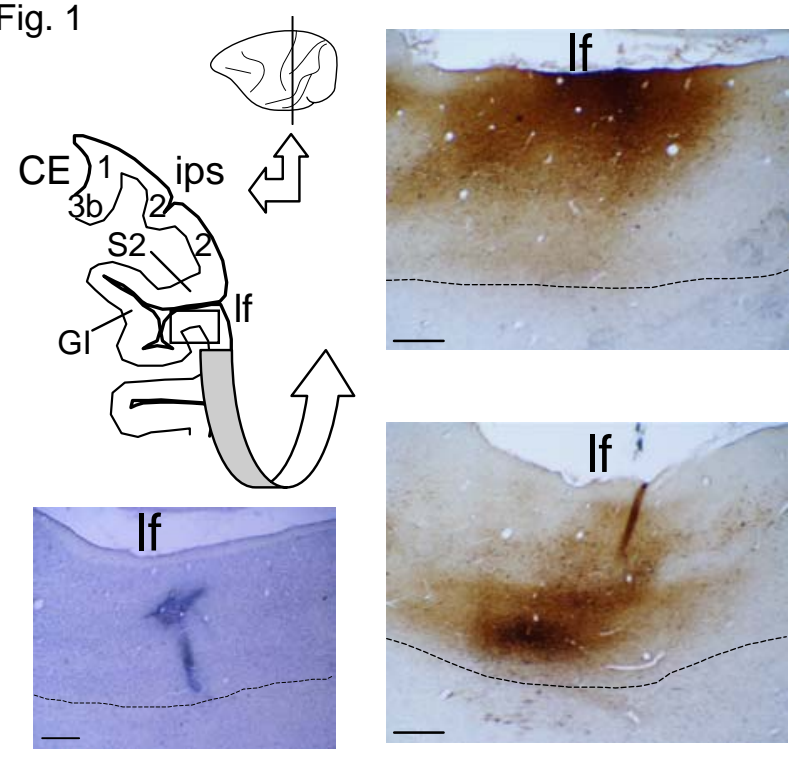


Fig. 2
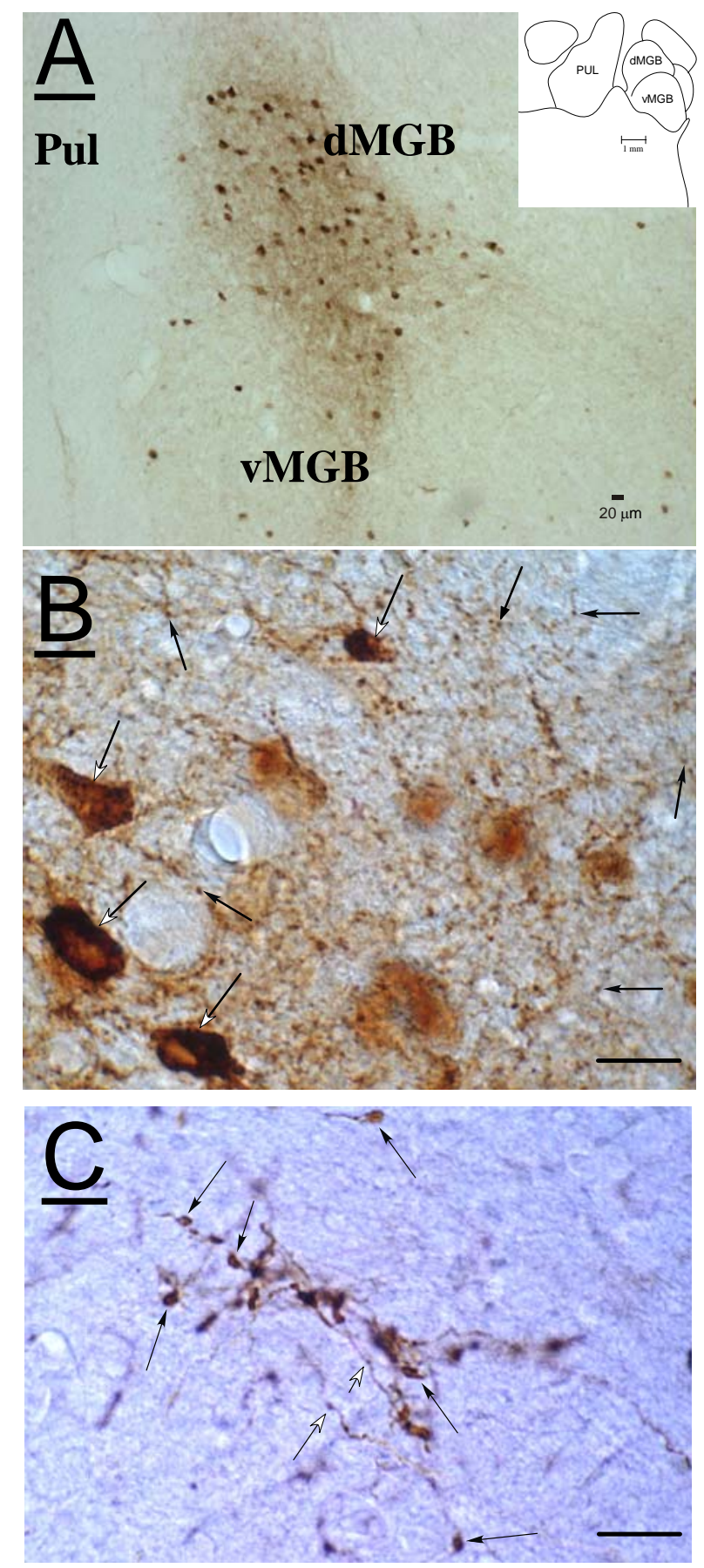
Fig. 3
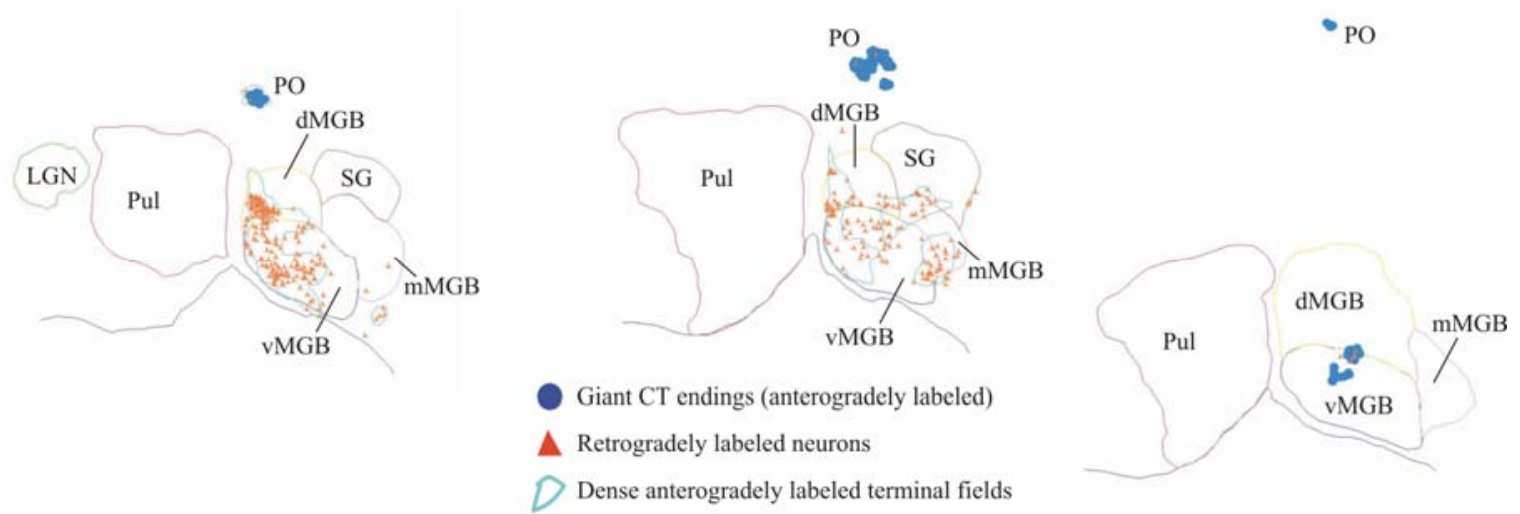